\title{
Dynamics of charged particles and magnetic dipoles around magnetized quasi-Schwarzschild black holes
}

\author{
Bakhtiyor Narzilloev ${ }^{1,2,3, a}$, Javlon Rayimbaev ${ }^{2,3,4,5, b}$, Ahmadjon Abdujabbarov $3,4,5,6,7, \mathrm{c}$, \\ Bobomurat Ahmedov ${ }^{3,5,7, \mathrm{~d}}$, Cosimo Bambi ${ }^{1, \mathrm{e}}$ \\ ${ }^{1}$ Center for Field Theory and Particle Physics and Department of Physics, Fudan University, Shanghai 200438, People's Republic of China \\ ${ }^{2}$ Akfa University, Kichik Halqa Yuli Street 17, 100095 Tashkent, Uzbekistan \\ ${ }^{3}$ Ulugh Beg Astronomical Institute, Astronomy Str. 33, 100052 Tashkent, Uzbekistan \\ ${ }^{4}$ Institute of Nuclear Physics, Ulugbek 1, 100214 Tashkent, Uzbekistan \\ ${ }^{5}$ National University of Uzbekistan, 100174 Tashkent, Uzbekistan \\ ${ }^{6}$ Shanghai Astronomical Observatory, 80 Nandan Road, Shanghai 200030, People's Republic of China \\ 7 Tashkent Institute of Irrigation and Agricultural Mechanization Engineers, Kori Niyoziy, 39, 100000 Tashkent, Uzbekistan
}

Received: 27 October 2020 / Accepted: 19 March 2021 / Published online: 31 March 2021

(c) The Author(s) 2021

\begin{abstract}
In the present paper, we have investigated the motion of charged particles together with magnetic dipoles to determine how well the spacetime deviation parameter $\epsilon$ and external uniform magnetic field can mimic the spin of a rotating Kerr black hole. Investigation of charged particle motion has shown that the deviation parameter $\epsilon$ in the absence of an external magnetic fields can mimic the rotation parameter of the Kerr spacetime up to $a / M \approx 0.5$. The combination of an external magnetic field and deviation parameter can do even a better job mimicking the rotation parameter up to $a / M \simeq 0.93$, which corresponds to the rapidly rotating case. Study of the dynamics of the magnetic dipoles around quasi-Schwarzschild black holes in the external magnetic field has shown that there are degeneracy values of the ISCO radius of test particles at $\epsilon_{c r}>\epsilon \geq 0.35$ which may lead to two different values of the innermost stable circular orbit (ISCO) radius. When the deviation parameter is in the range of $\epsilon \in(-1,1)$, it can mimic the spin of a rotating Kerr black hole in the range $a / M \in(0.0537,0.3952)$ for magnetic dipoles with values of the magnetic coupling parameter $\beta \in[-0.25,0.25]$ in corotating orbits.
\end{abstract}

\footnotetext{
a e-mail: nbakhtiyor18@fudan.edu.cn

b e-mail: javlon@astrin.uz

c e-mail: ahmadjon@ astrin.uz

d e-mail: ahmedov@astrin.uz

ee-mail: bambi@fudan.edu.cn (corresponding author)
}

\section{Introduction}

The first exact analytical solution of the vacuum field equations of Einstein's general relativity has been obtained just after its discovery in 1916 by Schwarzschild [1] and describes the exterior spacetime of the non-rotating spherically symmetric black hole. The rotating black hole solution was obtained by Kerr and includes two parameters: the total mass of the black hole and its rotation parameter. Most observational features of the astrophysical black holes can be, in principle, explained by the solution describing the Kerr black hole. On the other hand one may alternatively consider the extension of the Kerr solution with additional parameters, see, for example, [2-11]. The electric charge may also affect the gravitational field of the charged black hole and the properties of such objects have been studied in [12-18] for different astrophysical scenarios. The black hole may be considered as embedded on the brane of higher dimensional spacetime; see Refs. [19-23], where several properties of back holes with brane charge have been studied. Black holes may have gravitomagnetic monopole charge and the authors of Refs. [3,24-29] have studied the properties of spacetime with nonvanishing gravitomagnetic charge. The authors of Refs. [30-38] have studied the deformed spacetime of black holes and its properties.

One of the interesting extensions of the Kerr solution has been proposed in [4] where an approximate solution of the Einstein vacuum equations has been obtained. Also the leading order deviation from Kerr solution due to the spacetime quadrupole moment was introduced. The spacetime properties around the so-called quasi-Kerr black hole have been studied in Refs. [39,40]. In a previous paper, we have studied 
weak lensing near the quasi-Kerr black hole [41]. Recently we have also studied the charged particle motion around a quasi-Kerr compact object in the presence of a magnetic field [42].

Testing general relativity and alternative theories of gravity through gravitational lensing and motion of test particles under the various conditions is important to distinguish the central black hole parameters from the ones of alternate gravity theories, since their effects are similar or exactly the same at some range of values of the parameters of theories of gravity. For example in our previous work we have shown how the effects of the MOG field parameters [43], the conformal parameters [44,45], the coupling parameter of the EinsteinGauss-Bonnet theory [46], the electric charge of a black hole in Einstein-Maxwell theory [47], the stringy charge [45], perfect fluid dark matter [48] and quantum gravity [49] can mimic the spin of a rotating Kerr black hole. On the other hand, by now, in spite of the attempts to detect neutron stars as recycled radio pulsars near the supermassive black hole Sagittarius A* in the center of the Milky Way by the GRAVITY collaboration, we do not have any astrophysical observations of them. One of the reasons of the absence of the pulsars around $\mathrm{SgrA}^{*}$ is the scattering of radio waves in the plasma medium surrounding the SMBH and the other one might be the dominant effects of the magnetic interaction between the neutron star's dipole moment and the magnetic field around the black hole created by either the magnetic charge of the central black hole or the electric current of accreting matter. The stable circular and chaotic motions of neutral particles [50], the dynamics and quasiharmonic oscillations of charged particles around static and rotating black holes immersed in external asypmtotically uniform magnetic fields [51-55] and plasma magnetosphere surrounding black holes in different gravity models have been analyzed in detail by the authors of Refs. [56-60] in particular using the method of the Lyapunov to show the difference between regular and chaotic orbits. It is also shown that even a small misalignment and frame dragging effects cause a reduction of the chaotic motion.

Recent observation of the image of the supermassive black hole at the center of the elliptical galaxy M87 [61,62] and detection of the gravitational waves by the LIGO-Virgo collaboration $[63,64]$ provided a test of general relativity in the strong field regime. In fact gravitational waves generated by binary compact objects and the wave properties strongly depend on their spin and chaos degrees of freedom in the system. The existence of chaotic motion in such systems in the problems of two spinning black holes in the post-Newtonian approximation has been shown in Refs. [65-76].

At the same time these experiments and observations open a window for testing the modified and alternative theories of gravity together with an analysis of X-ray observations from active galactic nuclei (AGN) [77-79]. The second generation Very long baseline interferometer (VLBI) instrument
GRAVITY through precise observations of highly relativistic motions of matter and the $\mathrm{S} 2$ star close to Sgr A* has also provided experimental tests of general relativity in strong fields.

Despite the fact that in general relativity the black hole cannot have its own magnetic field due to the no-hair theorem [80], the latter can be considered as immersed in an external magnetic field [81] created by the current of electric charges in the accretion disk. The spacetime curvature will change the original structure of the external magnetic field. The detailed specifications of these changes and test particle motion around compact object in the presence of a magnetic field have been studied in Refs. [48,82-100]. The structure of the electromagnetic field around compact objects in alternate and modified theories of gravity has been explored in Refs. [45,53,101-122]. Quantum interference effects in conformal Weyl gravity have been studied in [123]. Periodic circular orbits, regular orbits and chaotic orbits of neutral and charged particles around various black holes have been investigated in Refs. [56-60,124, 125]. The magnetic dipole motion around a black hole in the presence of an asymptotically uniform magnetic field has been studied in $[126,127]$ with further development to the case of modified gravity theories in [43-45,49,128-135].

In this work our main purpose is to study the charged particles and magnetic dipoles motion around a magnetized quasiSchwarzschild black hole. The paper is organized as follows: Sect. 2 is devoted to a study of the dynamics of charged particles around a quasi-Schwarzschild black hole and comparison with one in Kerr spacetime. The magnetic dipole motion around a quasi-Schwarzschild black hole immersed in an external magnetic field is explored in Sect. 3. In this section the obtained results are compared with particles dynamics around Kerr black hole. Then the obtained results are applied to real astrophysical scenarios in Sect. 4. We conclude our results in Sect. 5. Throughout the paper we use the spacelike signature $(-,+,+,+)$ and the system of units where $G=1=c$.

\section{Charged particle motion: quasi-Schwarzschild versus Kerr black hole}

\subsection{Magnetic field around compact object}

Before going through the investigation of magnetized particles motion around a quasi-Schwarzschild compact object immersed in an external asymptotically uniform magnetic field we start with the case when the particle is electrically charged only. The quasi-Schwarzschild spacetime metric can be obtained from a rotating quasi-Kerr one [4] using the fol- 
lowing decomposition:

$g_{\mu \nu}=g_{\mu \nu}^{\mathrm{Schw}}+\epsilon h_{\mu \nu}$,

where $g_{\mu \nu}^{\text {Schw }}$ corresponds to the standard Schwarzschild metric and $\epsilon h_{\mu \nu}$ corresponds to the deviation from the Schwarzschild spacetime. The parameter $\epsilon$ defines the deviation from the spherically symmetric spacetime due to the additional term in the mass quadrupole moment $Q$ of the gravitating object by

$Q=-\epsilon M^{3}$,

and it can take both negative and positive signs [4]. In the linear approximation in $\epsilon$ the contravariant components of the spacetime metric can be written as

$g^{\mu \nu}=g_{S c h w}^{\mu \nu}-\epsilon h^{\mu v}$

and thus the upper indices of $h^{\mu \nu}$ can be lowered by using the Schwarzschild metric tensor. The contravariant components of $h^{\mu \nu}$ are given by the expressions (see [4])

$h^{t t}=f^{-1}\left(1-3 \cos ^{2} \theta\right) F_{1}$,

$h^{r r}=f\left(1-3 \cos ^{2} \theta\right) F_{1}$,

$h^{\theta \theta}=-\frac{1}{r^{2}}\left(1-3 \cos ^{2} \theta\right) F_{2}$,

$h^{\phi \phi}=-\frac{1}{r^{2} \sin ^{2} \theta}\left(1-3 \cos ^{2} \theta\right) F_{2}$,

$f=1-\frac{2 M}{r}$

where the radial functions $F_{1}$ and $F_{2}$ read

$$
\begin{aligned}
F_{1}= & -\frac{5(r-M)\left(2 M^{2}+6 M r-3 r^{2}\right)}{8 M r(r-2 M)} \\
& -\frac{15 r(r-2 M) \log \left(\frac{r}{r-2 M}\right)}{16 M^{2}}, \\
F_{2}= & \frac{5\left(2 M^{2}-3 M r-3 r^{2}\right)}{8 M r} \\
& +\frac{15\left(r^{2}-2 M^{2}\right) \log \left(\frac{r}{r-2 M}\right)}{16 M^{2}} .
\end{aligned}
$$

After lowering the indices of $h^{\mu \nu}$ with the use of $g_{\mu \nu}^{\text {Schw }}$ the quasi-Schwarzschild spacetime metric takes the following form:

$\mathrm{d} s^{2}=g_{t t} \mathrm{~d} t^{2}+g_{r r} \mathrm{~d} r^{2}+k(r, \theta) r^{2} \mathrm{~d} \Omega^{2}$,

where

$$
\begin{aligned}
g_{t t} & =-f\left[1-\epsilon F_{1}\left(1-3 \cos ^{2} \theta\right)\right], \\
g_{r r} & =f^{-1}\left[1+\epsilon F_{1}\left(1-3 \cos ^{2} \theta\right)\right], \\
k(r, \theta) & =1-\epsilon F_{2}\left(1-3 \cos ^{2} \theta\right) .
\end{aligned}
$$

In the spacetime metric the terms being proportional to $\epsilon$ provide the part being responsible for the quasi-Schwarzschild effects. One can easily check that in the case of $\epsilon=0$ one recovers Schwarzschild spacetime. It is worth noting here that the condition $g^{r r}=0$ gives the location of an event horizon at $r_{e}=2 M$, being the same as in the case of the Schwarzschild black hole.

Using the Wald method [81] one can find the components of the four-vector potential of electromagnetic fields as

$A^{\mu}=\left(0,0,0, \frac{1}{2} B\right)$.

Using the metric (3) one can write the covariant components as

$A_{\mu}=\left\{0,0,0, \frac{1}{2} B h(r, \theta) r^{2} \sin ^{2} \theta\right\}$.

Now one can find the expression for the magnetic field around a quasi-Schwarzschild compact object. The four-velocity of the proper observer is given by

$\mathcal{U}^{\alpha}=\left\{\left(f\left[1+\frac{1}{2} \epsilon F_{1}(1+3 \cos 2 \theta)\right]\right)^{-\frac{1}{2}}, 0,0,0\right\}$.

Then the orthonormal components of the magnetic field with respect to the chosen frame takes the following form [42]:

$$
\begin{aligned}
B^{\hat{r}}= & B \cos \theta \frac{1+\epsilon F_{2}(3 \cos 2 \theta-1)}{1+\frac{\epsilon}{2} F_{2}(3 \cos 2 \theta+1)}, \\
B^{\hat{\theta}}= & B \sin \theta \sqrt{f \frac{\sqrt{1+\frac{\epsilon}{2} F_{1}(1+3 \cos 2 \theta)}}{\sqrt{1+\frac{\epsilon}{2} F_{2}(3 \cos 2 \theta+1)}}} \\
& \times \frac{1+\frac{\epsilon}{4}\left[r F_{2}^{\prime}+2 F_{2}\right](3 \cos 2 \theta+1)}{\sqrt{1-\frac{\epsilon^{2}}{4} F_{1}^{2}(3 \cos 2 \theta+1)^{2}}}, \\
B^{\hat{\phi}}= & 0,
\end{aligned}
$$

where the prime' denotes the derivative with respect to the radial coordinates. One can easily see that in pure Schwarzschild spacetime it takes the form

$B^{\hat{r}}=B \cos \theta$
$B^{\hat{\theta}}=B \sqrt{f} \sin \theta$

and in the Newtonian weak field regime $M / r \rightarrow 0$ the components of the magnetic field become

$B^{\hat{r}}=B \cos \theta, \quad B^{\hat{\theta}}=B \sin \theta$,

consistent with the Newtonian limit. 

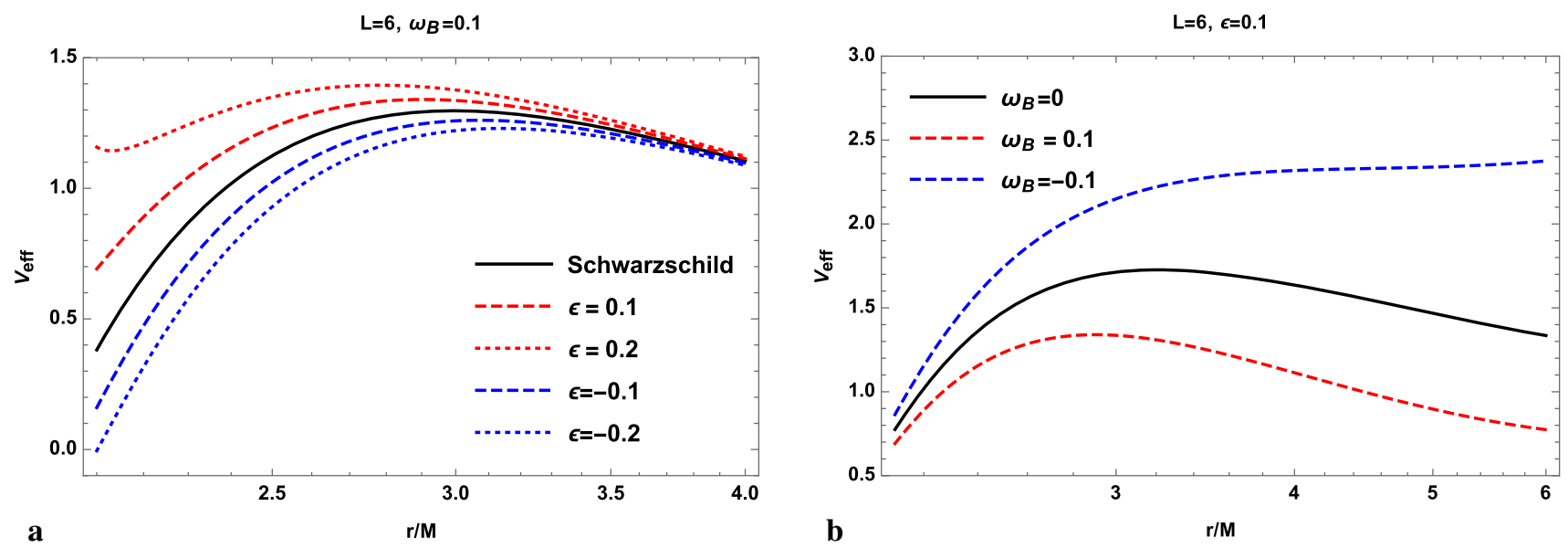

Fig. 1 Effective potential as a function of radial coordinate $r$ for the case $M=1$. The left panel corresponds for the variation of the deformation parameter. The right panel is for the variation of the magnetic interaction

\subsection{Circular motion of charged test particle around a quasi-Schwarzschild compact object}

Now, we briefly study the equation of motion of a charged particle around a quasi-Schwarzschild compact object. It is more convenient to use the Hamilton-Jacobi equation of motion for particles orbiting around central objects, which is the case here. In the presence of an external electromagnetic field the equation reads

$g^{\alpha \beta}\left(\frac{\partial S}{\partial x^{\alpha}}+e A_{\alpha}\right)\left(\frac{\partial S}{\partial x^{\beta}}+e A_{\beta}\right)=-m^{2}$,

with $e$ and $m$ being the electric charge and mass of the test particle, respectively.

The equation of motion (16) is not separable when the system is non-integrable. In this case, Eq. (16) should be replaced with a Hamiltonian formalism [51,56-60]. However, when one investigates test particle motion in the equatorial plane, Eq. (16) can be expressed in the following separable form:

$S=-E t+L \phi+S_{r}+S_{\theta}$,

where $E$ and $L$ define the energy and angular momentum of a test particle per unit mass, respectively. Thus, the equation of motion of a test particle with unit mass reads

$$
\begin{aligned}
& \frac{\left(\mathcal{L}+\frac{e B}{2 m} r^{2}\left(1-\epsilon F_{2}\right)\right)^{2}}{r^{2}\left(1-\epsilon F_{2}\right)}+\frac{f}{1+\epsilon F_{1}}\left(\frac{\partial S}{\partial r}\right)^{2} \\
& -\frac{\mathcal{E}^{2}}{\left.f\left(1-\epsilon F_{1}\right)\right)}=-1
\end{aligned}
$$

where $\mathcal{E}=E / m$ and $\mathcal{L}=L / m$ are the specific energy and angular momentum of a test particle, respectively.
For test particles moving at the equatorial plane $(\theta=\pi / 2)$ one can obtain the effective potential from the radial motion

$\dot{r}^{2}=\mathcal{E}^{2}-V_{\text {eff }}$,

which reads

$V_{\mathrm{eff}}=f\left(1-F_{1} \epsilon\right)\left\{1+\left(\frac{\mathcal{L}}{h\left(r, \frac{\pi}{2}\right) r^{2}}-\omega_{B}\right)^{2}\right\}$,

with $\omega_{B}=e B /(2 m c)$ defining the cyclotron frequency of a charged particle which corresponds to the interaction between electrically charged particle and external magnetic field. The radial dependence of such an effective potential is shown in Fig. 1. It is clearly seen that the increase in the deviation parameter also increases the effective potential while the magnetic field has an opposite action as was shown in Refs. [51-53]

For a circular motion of a particle at equatorial plane one can set the following standard conditions:

$V_{\text {eff }}(r)=\mathcal{E}^{2}, V_{\text {eff }}^{\prime}(r)=0$,

which results in the angular momentum of a charged test particle to have the radial dependence as presented in Fig. 2. We see the usual Schwarzschild shape for the line for which $\epsilon=0$ in the right panel. However, starting from some value of the deviation parameter around $\epsilon \approx 0.32$ it changes the shape of lines, which causes them to have a maximum point at the corresponding radius that vanishes starting from the case when $\epsilon \approx 0.8$. We will come back to this point later in the next subsection where it contains an important description of defining the ISCO radius. 
$\epsilon=0.2$

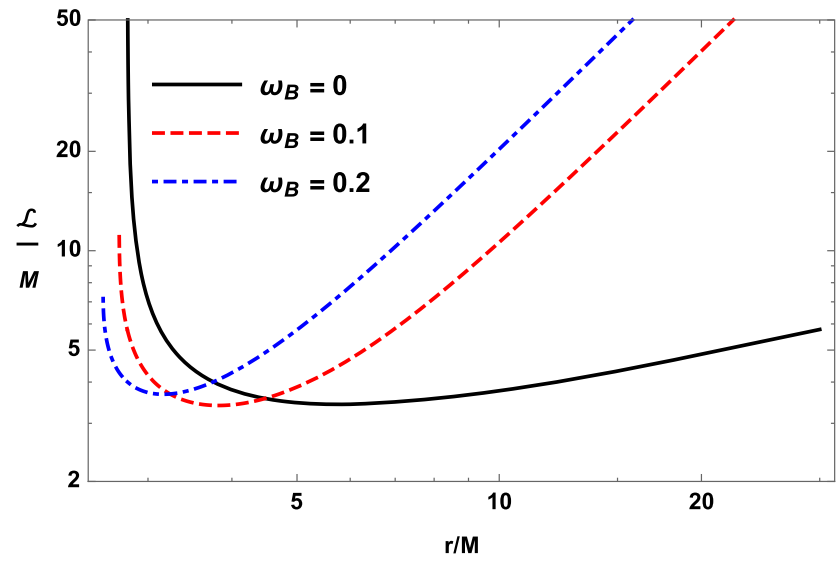

$\omega_{B}=0$

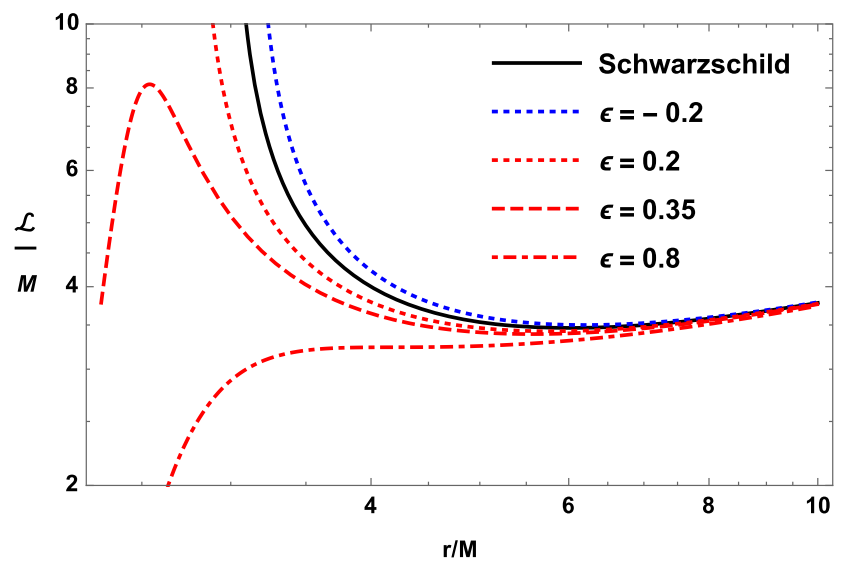

Fig. 2 Radial dependence of the angular momentum of a test particle for the different values of the magnetic interaction (left panel) and deformation (right panel)

\subsection{Innermost stable circular orbits: quasi-Schwarzschild versus Kerr black hole}

In this subsection we investigate so-called innermost stable circular orbits (ISCO) around a quasi-Schwarzschild compact object immersed in an asymptotically uniform magnetic field. Based on the obtained results we will try to answer the question how the parameters $\epsilon$ and $B$ can mimic the rotation parameter $a$ of the well-known Kerr solution. The idea is that, if the parameters mentioned can mimic the rotation parameter $a$ of a Kerr black hole, then for the same ISCO radius one can get a correspondence constraint between the rotation parameter and the parameters $\epsilon$ and $B$. First, we investigate the relation between the ISCO radius and the parameters of interest. To do so, one can add additional requirement to the condition (21), reading

$V_{\mathrm{eff}}^{\prime \prime}(r)=0$.

Taking into account these three conditions (together with (21)) one might plot the mutual dependence between the ISCO radius and parameters $\epsilon$ and $B$ as plotted in Fig. 3 . One can see from the graphs that the increase of the two parameters $\epsilon$ and $B$ reduces the ISCO radius of a charged test particle. From the left panel it is clearly seen that if one increases the deviation parameter $\epsilon$ up to some value then the ISCO radius starts to reduce, becoming smaller and smaller instead of taking its initial values before reducing. To make the situation clear one should take into account the condition on angular momentum of a test particle that says that for a particle to move on the last stable circular orbit its angular momentum should have a minimum on that orbit radius. Using this condition one can refer to the graph of dependence between angular momentum of a test particle on circular orbit radius as in the right panel of Fig. 2. We have mentioned this point in the previous subsection saying that the angular momentum for the absence of external magnetic field would have maximum points when the deviation parameter is between 0.32 and 0.8 . But, plotting Fig. 3 we just used the case when the angular momentum of a test particle has an extremum. So, we need to exclude such maxima from these extremum points, which results in the fact that one needs to erase the lower part of ISCO lines starting from the turning points, which is shown with shaded region in the left panel of Fig. 3. For the case of a magnetic field the situation is typical as expected, i.e. if one increases the magnetic field the Lorentz force becomes stronger, which makes the ISCO radius smaller.

Finally, we plan to answer the question stated in the beginning of this subsection: how well can the parameters $\epsilon$ and $B$ mimic the rotation parameter $a$ of the Kerr metric? Knowing how the ISCO in the case of Kerr metric behaves under the influence of a rotation parameter one can plot the degeneracy between these parameters as shown in Fig. 4. It was expected from the dependence of ISCO on the parameter $\epsilon$ that this parameter cannot completely mimic the rotation parameter as the ISCO radius did not tend to $M$, which is the case for an extremal rotation $a \rightarrow 1$ in the Kerr metric. Now it is once more clearly seen that this parameter can only mimic the rotation parameter up to approximately $\approx 0.5$ when the external magnetic field is absent. One can, however, see that the magnetic parameter itself can mimic the rotation parameter up to $\approx 0.88$. It is also seen from the right panel that in the presence of both magnetic field and deviation parameter the mimic range exceeds $a>0.9$, being competitive with the rapidly rotating Kerr spacetime.

The degeneracy plot between the deviation parameter $\epsilon$ and the magnetic coupling parameter $\omega_{B}$ for a few fixed values of the ISCO radius is illustrated in Fig. 5. We see that the magnetic interaction has a considerably stronger effect than the effect of deformation of a spacetime. 

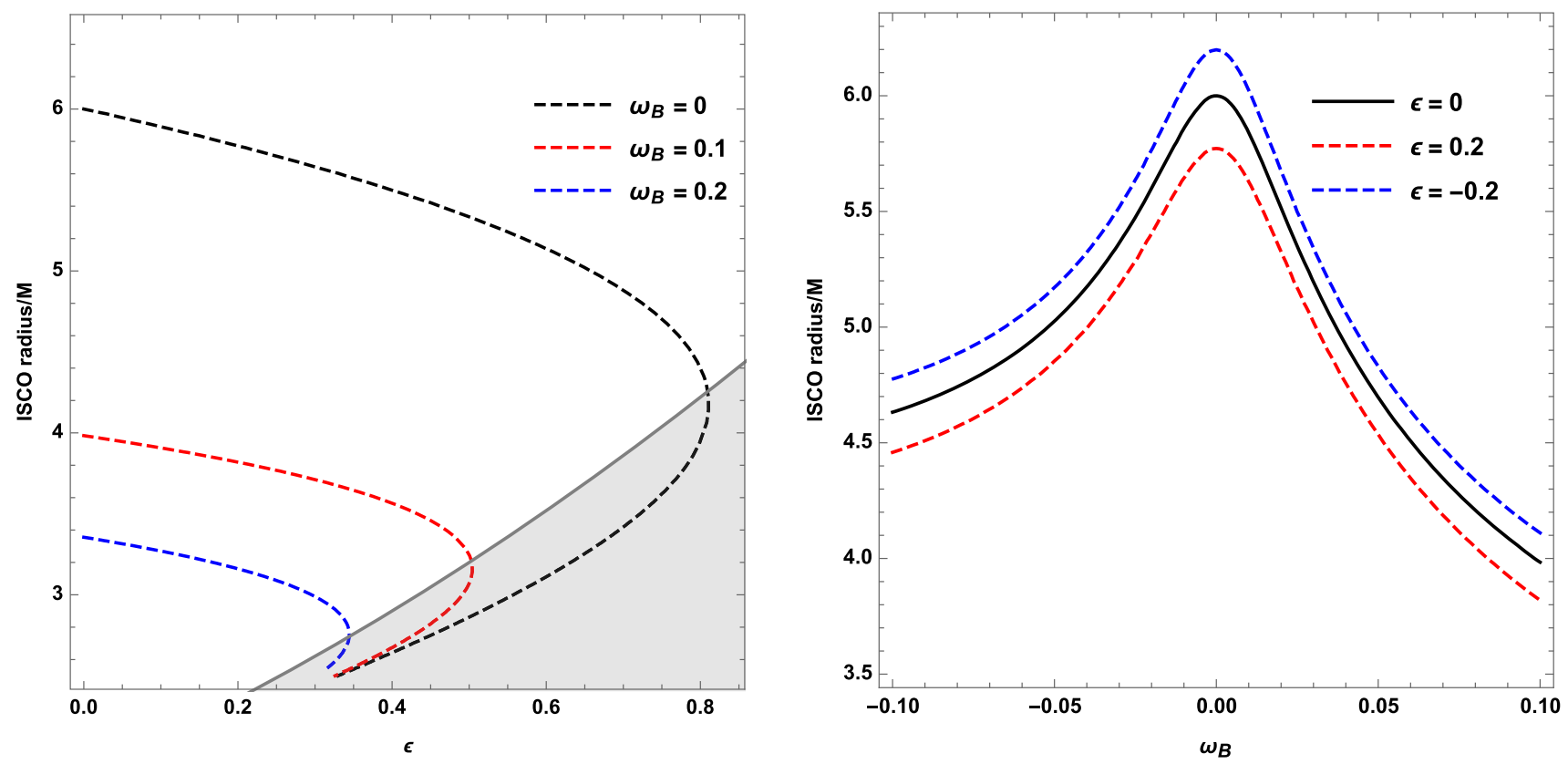

Fig. 3 ISCO radius of a test particle orbiting in the equatorial plane of a quasi-Schwarzschild compact object for different values of the magnetic interaction (left panel) and deformation parameter (right panel)
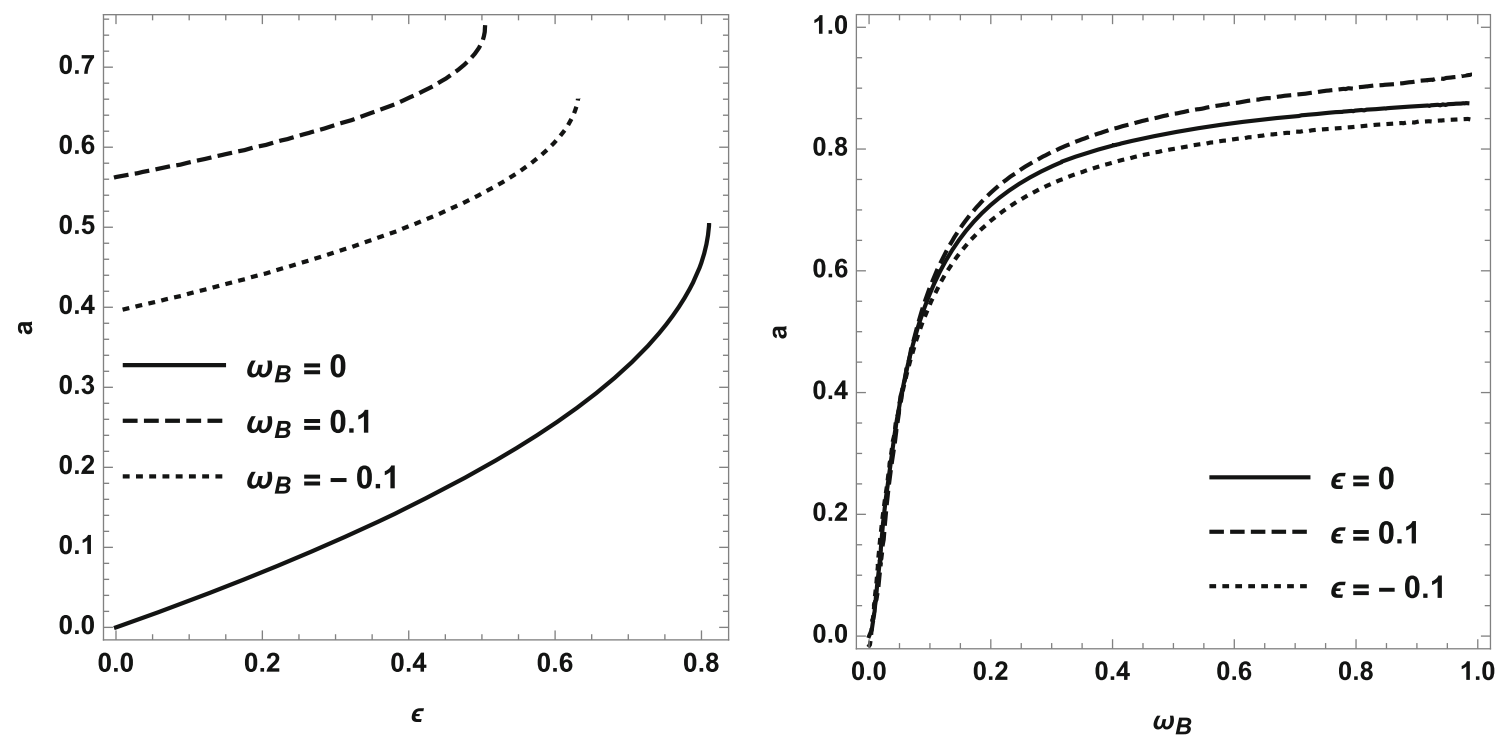

Fig. 4 Degeneracy plot that shows the correspondence between of the dimensionless rotation parameter $a$ of a Kerr metric with $\epsilon$ and $B$ parameters

\section{Magnetic dipole motion: quasi-Schwarzschild versus Kerr black hole}

In this section we focus on the magnetic dipole motion around a quasi-Schwarzschild compact object immersed in an asymptotically uniform magnetic field. The HamiltonJacobi equation of motion of magnetic dipole takes the following form:

$g^{\mu \nu} \frac{\partial \mathcal{S}}{\partial x^{\mu}} \frac{\partial \mathcal{S}}{\partial x^{\nu}}=-\left(m-\frac{1}{2} D^{\mu \nu} F_{\mu \nu}\right)^{2}$
Here, $D^{\mu \nu}$ is the antisymmetric polarization tensor which defines the electrodynamic properties of the particle. We assume that the particle is electrically neutral $q=0$ and the polarization tensor is only described by the magnetic moment $\mu$ itself. It is worth to note here that it is also possible to investigate in an alternate way the motion of the magnetic dipole with an electric charge where one just needs to take the left hand side of Eq. (16) instead of the one in Eq. (23). However, in this work we aim to apply the motion of the magnetic dipoles to magnetized neutron stars orbiting 


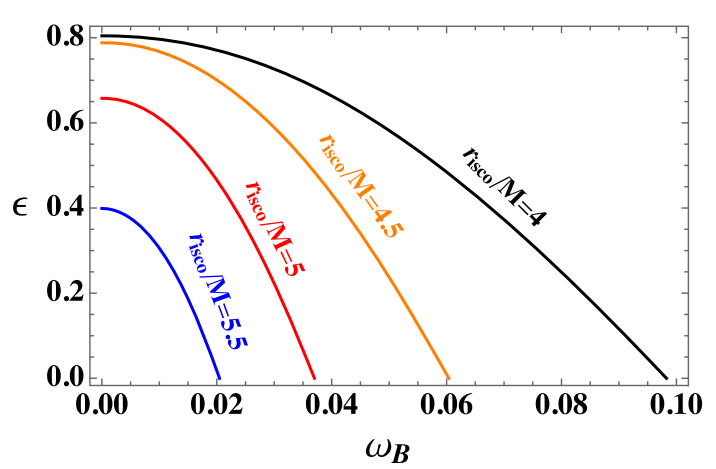

Fig. 5 Degeneracy plots for $\epsilon$ and $\omega_{B}$ for given values of ISCO radii

around supermassive black holes where the neutron star can be treated as an electrically neutral test particle with nonvanishing magnetic dipole moment. Since the mass of a typical supermassive black hole is much greater than the mass of a typical neutron star this allows us to take the neutron star as a test particle moving in the spacetime of the former one. Therefore hereafter we focus on the motion of electrically neutral magnetic dipole only. In this case the components of this tensor can be written as [45]

$D^{\mu \nu}=\eta^{\mu \nu \alpha \beta} u_{\alpha} \mu_{\beta}$,

which satisfies the following condition:

$D^{\mu v} u_{v}=0$,

where $\mu_{\alpha}$ describes the magnetic four-momentum of a magnetic dipole. $F_{\mu \nu}=\partial_{\mu} A_{\nu}-\partial_{\nu} A_{\mu}$ is the electromagnetic field tensor, which can also be written in terms of the components of electromagnetic field as

$F_{\mu \nu}=-\eta_{\mu \nu \alpha \beta} B^{\alpha} u^{\beta}+2 u_{[\mu} E_{\nu]}$

where $\eta_{\alpha \beta \sigma \gamma}$ is the pseudo-tensorial form of the Levi-Civita symbol $\epsilon_{\alpha \beta \sigma \gamma}$ defined as

$\eta_{\alpha \beta \sigma \gamma}=\sqrt{-g} \epsilon_{\alpha \beta \sigma \gamma}, \quad \eta^{\alpha \beta \sigma \gamma}=-\frac{1}{\sqrt{-g}} \epsilon^{\alpha \beta \sigma \gamma}$,

with $g=\operatorname{det}\left|g_{\mu \nu}\right|=-r^{4} \sin ^{2} \theta$ for the spacetime metric (3) and

$\epsilon_{\alpha \beta \sigma \gamma}=\left\{\begin{array}{c}+1, \text { for even permutations }, \\ -1, \text { for odd permutations }, \\ 0, \text { for the other combinations } .\end{array}\right.$

Having taken the contraction with (24) and using (25) with (26) one can write

$D^{\mu \nu} F_{\mu \nu}=2 \mu^{\alpha} B_{\alpha}=2 \mu^{\hat{\alpha}} B_{\hat{\alpha}}$.
For simplicity we consider the magnetic interaction between the magnetic dipole and external magnetic field to be weak enough (due to the weakness of the external test magnetic field), so we can use the approximation $\left(\mathcal{D}^{\mu \nu} \mathcal{F}_{\mu \nu}\right)^{2} \rightarrow 0$. It is expected that in a given external magnetic field the magnetic momentum of a particle aligns along this external field. If one assumes a particle moving at the equatorial plane $(\theta=\pi / 2)$ then, since this magnetic field has only a normal component $B^{\hat{\theta}}$ to this equatorial plane, so does the magnetic moment $\mu^{\hat{\theta}}$, which is consistent with the lowest energy condition of the magnetic dipole. The scalar product (29) then becomes

$D^{\mu \nu} F_{\mu \nu}=2 \mu B \mathcal{A} ;$

here $\mathcal{A}(r)$ defines the proportionality function taking into account Eq. (10) and for $B^{\hat{\theta}}$ reads

$\mathcal{A}=\mathcal{A}(r)=\sqrt{f \frac{1-\epsilon F_{1}}{1-\epsilon F_{2}}} \frac{1-\frac{\epsilon}{2}\left[r F_{2}^{\prime}+2 F_{2}\right]}{\sqrt{1-\epsilon^{2} F_{1}^{2}}}$.

Plugging the scalar product of $D^{\mu v}$ and $F^{\mu v}$ into the equation of motion (23) one can find the effective potential at the equatorial plane:

$V_{\text {eff }}=\left(1-\frac{2 M}{r}\right)\left[1-\epsilon F_{1}\right]\left\{(1-\beta \mathcal{A})^{2}+\frac{\mathcal{L}^{2}}{r^{2}\left[1-\epsilon F_{2}\right]}\right\}$,

here $\beta=2 \mu B / m$ is called the magnetic coupling parameter; it defines the electromagnetic interaction between magnetic dipole and external magnetic field. In real astrophysical scenarios, for example in the case of a typical neutron star orbiting around a supermassive black hole with magnetic dipole moment $\mu=(1 / 2) B_{\mathrm{NS}} R_{\mathrm{NS}}^{3}$ the coupling magnetic parameter is

$\beta \simeq \frac{11}{250}\left(\frac{B_{\mathrm{NS}}}{10^{12} \mathrm{G}}\right)\left(\frac{R_{\mathrm{NS}}}{10^{6} \mathrm{~cm}}\right)^{3}\left(\frac{B_{\mathrm{ext}}}{10 \mathrm{G}}\right)\left(\frac{m_{\mathrm{NS}}}{M_{\odot}}\right)^{-1}$,

where $B_{\mathrm{NS}}$ is the magnetic field at the surface of the neutron star, $R_{\mathrm{NS}}$ and $m_{\mathrm{NS}}$ are radius and mass of the neutron star, respectively. The radial dependence of the effective potential is plotted in Fig. 6. We see that effective potential for the magnetic dipole behaves similarly to the charged particle one.

From the same conditions (21) as for the trajectory of the particle to be circular one can easily find the expressions for the angular momentum and the energy of the test particle: 


$$
\begin{aligned}
& \mathcal{L}^{2}=\frac{r^{3}(1-\mathcal{A} \beta)\left(1-\epsilon F_{2}\right)^{2}\left\{(1-\mathcal{A} \beta)\left[f^{\prime}\left(1-\epsilon F_{1}\right)-\epsilon f F_{1}^{\prime}\right]-2 \beta f \mathcal{A}^{\prime}\left(1-\epsilon F_{1}\right)\right\}}{\left(1-\epsilon F_{2}\right)\left[\left(1-\epsilon F_{1}\right)\left(2 f-r f^{\prime}\right)+f r \epsilon F_{1}^{\prime}\right]-f r \epsilon\left(1-\epsilon F_{1}\right) F_{2}^{\prime}}, \\
& \mathcal{E}^{2}=\frac{(1-\beta \mathcal{A}) f^{2}\left(1-\epsilon F_{1}\right)^{2}\left\{(1-\beta \mathcal{A}) r \epsilon F_{2}^{\prime}+2\left(1-\epsilon F_{2}\right)\left[1-\beta\left(\mathcal{A}+r \mathcal{A}^{\prime}\right)\right]\right\}}{\left(1-\epsilon F_{2}\right)\left[\left(1-\epsilon F_{1}\right)\left(2 f-r f^{\prime}\right)+\epsilon f r F_{1}^{\prime}\right]-f r \epsilon\left(1-\epsilon F_{1}\right) F_{2}^{\prime}} .
\end{aligned}
$$

The orbit of a particle moving at equatorial plane being circular makes the angular momentum of a particle have the radial dependence as plotted in Fig. 7. From the shift of the minimum of the lines one can state how the ISCO radius changes for the different values of the magnetic coupling parameter and also for various values of the deviation parameter. From the upper panel it appears that if one increases the magnetic interaction between magnetic dipole and external magnetic field then the ISCO radius increases.

Having obtained the effective potential and the radial dependence for the angular momentum one can now investigate ISCO for a magnetic dipole moving at the equatorial plane. We can use either the condition given in Eq. (22) or the angular momentum to have a minimum at the ISCO radius. Then the dependence of the ISCO radius of the parameter $\epsilon$ and the magnetic coupling parameter $\beta$ becomes as presented in Fig. 8.

Figure 8 demonstrates the dependence of the ISCO radius of magnetic dipole around a quasi-Schwarzschild black hole from the deviation (on the top panel) and magnetic coupling (on the bottom panel) parameters. We see from the plots that increasing the magnetic coupling parameter increases the ISCO radius. Moreover, it appears that it has a value around $\beta=2 / 3$ at which the ISCO radius tends to infinity, telling that no stable circular orbits can occur no matter how far the magnetic dipole is orbiting. In the upper panel we have cut the lower part of the ISCO radius dependence for the same reason as in the previous section which tells that for the chosen value of the deviation parameter the angular momentum of a test particle can have both a minimum and a maximum where we should take only the minimum points that are physically relevant.

\section{Astrophysical applications of the study}

One of the important and actual key issues in relativistic astrophysics is testing the theory of gravity by a study of the test magnetic dipoles motion, in particular, in an exploration of the motion of the neutron stars (pulsars and/or magnetars) treated as test magnetic dipoles around supermassive black holes (hereafter SMBH), which may give a possibility to test both gravitational and electromagnetic fields around a SMBH due to their accurate pulses in observations which may help to measure the distance through Doppler effect. In the observations of such models it can be realized that the neutron star could be found near a galactic center. However, by now, it is quite difficult to find radio pulsars, due to Compton scattering of radio pulses in a dense charged electron gas around the Sgr A*. The first and by now a single neutron star-magnetar, called SGR 1745-2900, around Sgr A* has been discovered in 2013 [136]. In our calculations we use the parameters of the magnetar treating it as a magnetic dipole orbiting Sgr A*. On other hand, theoretical problematic issues occur on the analysis of observational properties, such as QPO, the ISCO radius around the SMBH when the parameters of different alternate gravity reflect similar effects on the properties; in those cases it is impossible for gravity's effect to play a dominant role. In fact mostly astrophysical black holes are accepted as rotating black holes. Here we aimed to analyze the ISCO radius, comparing it with the effects of spacetime deformation and spin of a Kerr black hole when the two provide the same value for the ISCO radius of the magnetic dipoles. Note that for comparison we consider a quasi-Schwarzschild compact object immersed in an external asymptotically uniform magnetic field and a Kerr black hole without magnetic field. We assume the real astrophysical case of the magnetar orbiting the SMBH Sgr A*. Note also that one can consider a magnetic dipole as a neutral one in the absence of an external magnetic field.

The value of the magnetic coupling parameter $\beta$ for the magnetar SGR (PSR) J1745-2900 with the magnetic dipole moment $\mu \simeq 1.6 \times 10^{32} \mathrm{G} \cdot \mathrm{cm}^{3}$ orbiting the supermassive BH Sgr $\mathrm{A} *$ is [136]

$\beta_{\text {PSR J1745-2900 }} \simeq 0.716\left(\frac{B_{\text {ext }}}{10 \mathrm{G}}\right)$.

The ISCO radius of test particles around a rotating Kerr black hole is defined by the following expression for retrograde $(+)$ and prograde $(-)$ orbits [137]:

$r_{\text {isco }}=3+Z_{2} \pm \sqrt{\left(3-Z_{1}\right)\left(3+Z_{1}+2 Z_{2}\right)}$,

where

$Z_{1}-1=(\sqrt[3]{1+a}+\sqrt[3]{1-a}) \sqrt[3]{1-a^{2}}, \quad Z_{2}^{2}-Z_{1}^{2}=3 a^{2}$ 
$L=6, \beta=0.2$

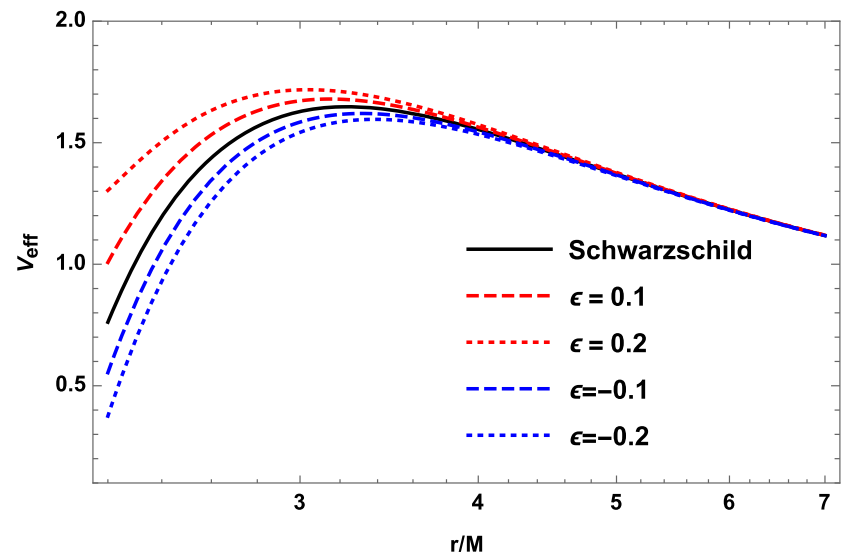

$L=6, \epsilon=0.1$

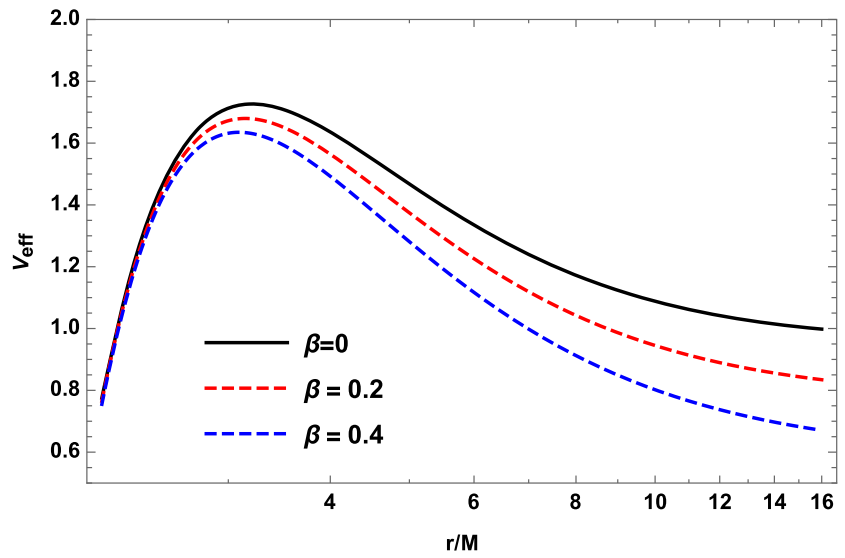

Fig. 6 Effective potential as a function of $r / M$ for different values of the deviation (left panel) and the magnetic interaction (right panel) parameters
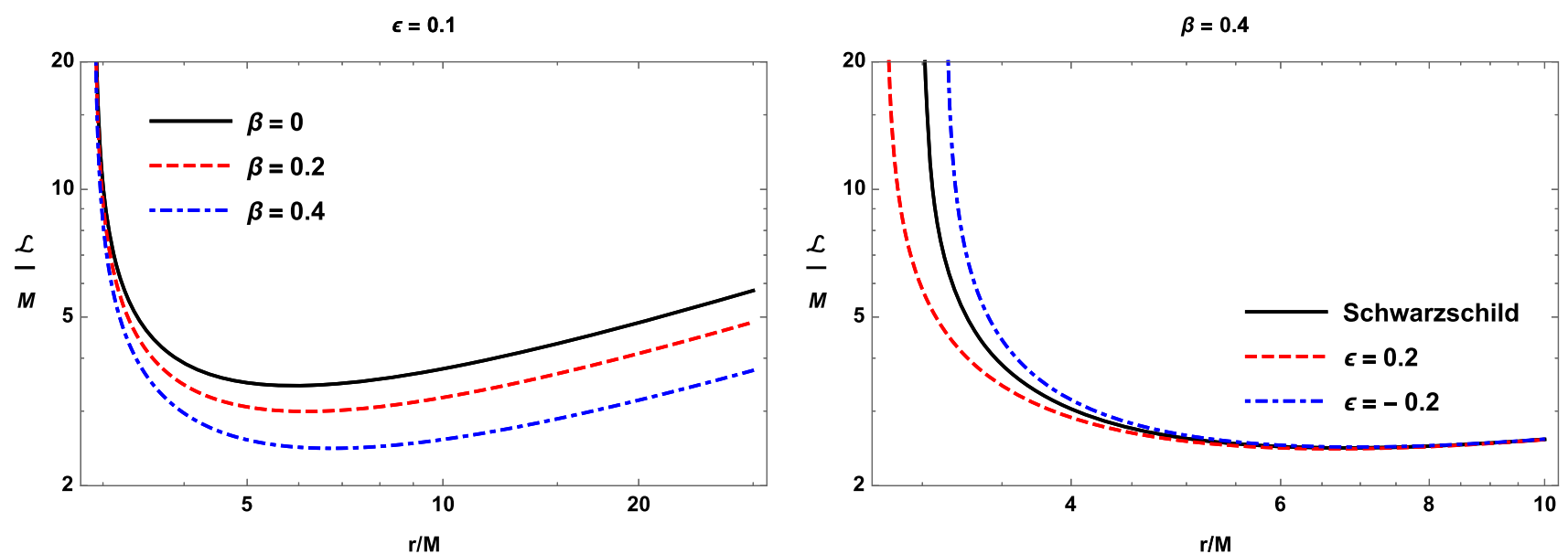

Fig. 7 Radial dependence of the angular momentum of a magnetic dipole for the different values of the magnetic parameter $\beta$ (left panel) and deviation $\epsilon$ (right panel)
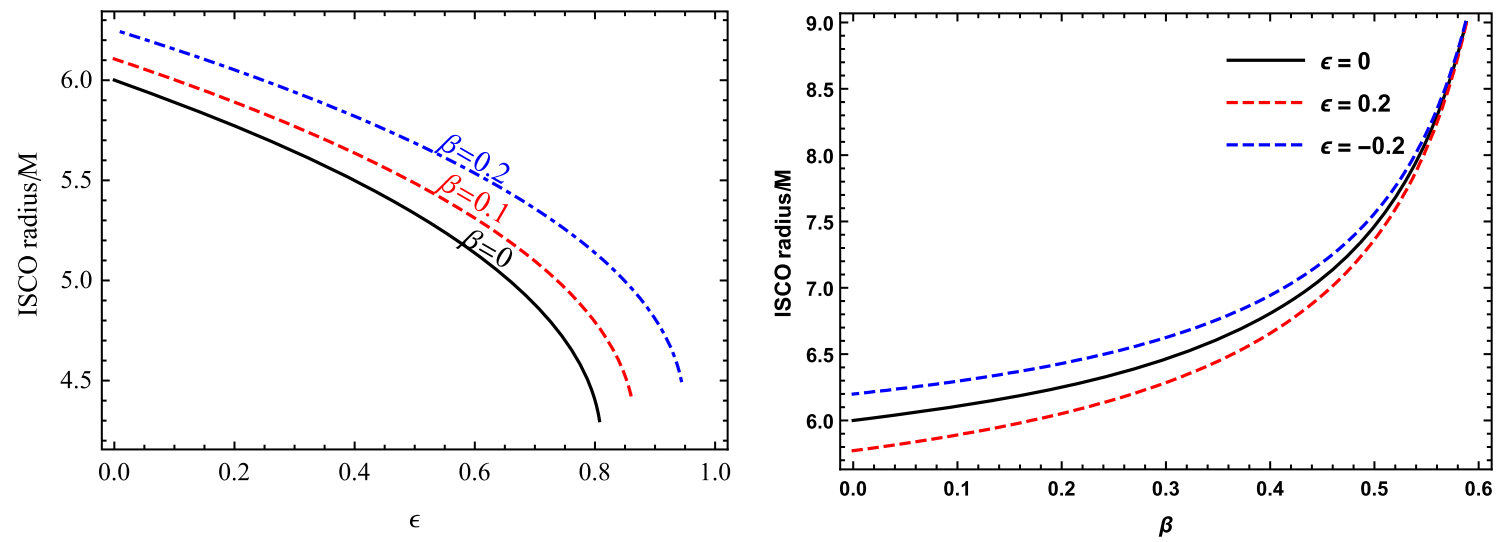

Fig. 8 Dependence of the ISCO radius of test particles orbiting at the equatorial plane around a quasi-Schwarzschild black hole from the deviation for given $\beta$ (left panel) and magnetic coupling parameters for given $\epsilon$ (right panel) 


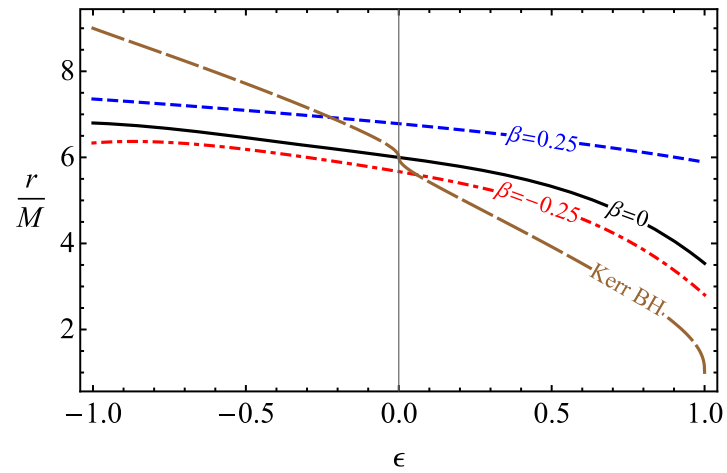

Fig. 9 Dependence of the ISCO radius of the magnetic dipoles from the deviation parameter of a quasi-Schwarzschild black hole and the spin of a Kerr black hole (left panel). The degeneracy plot is for the

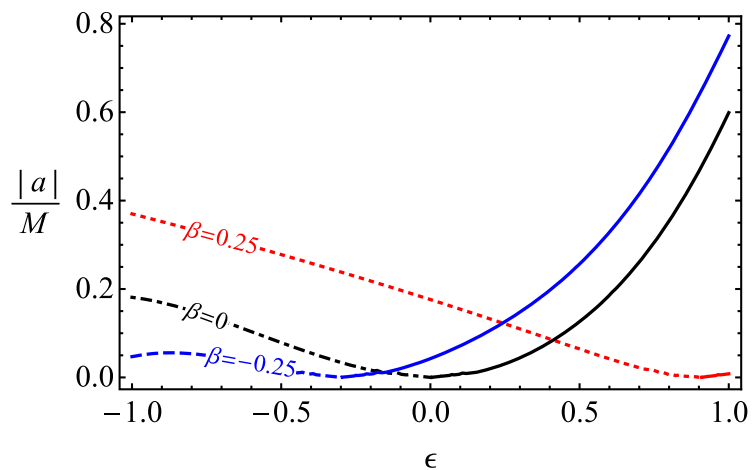

spin of a Kerr BHs $a$ and the deviation parameter $\epsilon$ for the different values of the magnetic coupling parameter $\beta=0.25,0,-0.25$

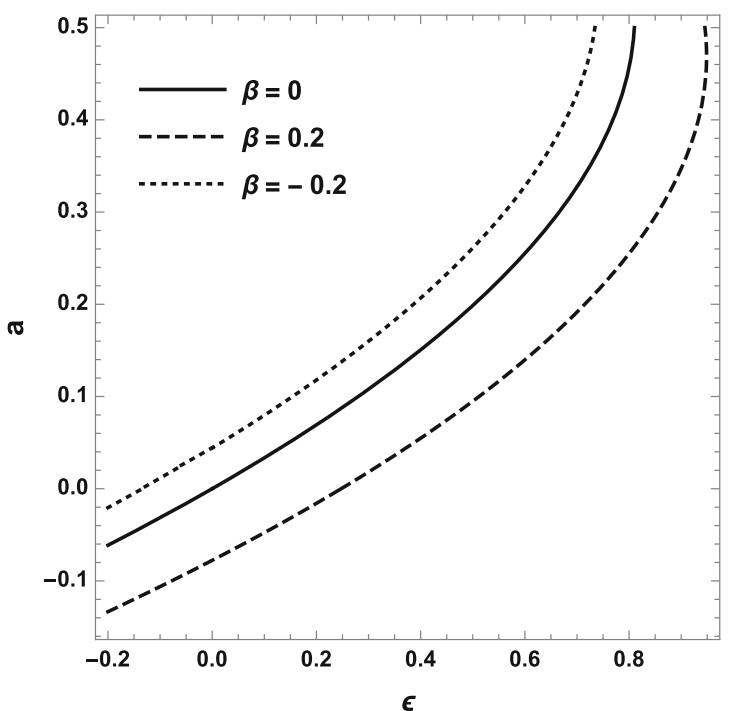

Fig. 10 Degeneracy plot between dimensionless spin of the Kerr BHs $a$ and the magnetic coupling parameter $\beta$ and deviation parameter $\epsilon$ providing the same value for the ISCO radius for magnetic dipoles around the Schwarzschild black hole immersed in an external asymptotically uniform magnetic field

Now, in order to compare the effects of the spin and deviation parameters on the ISCO radius we will present the dependence of the ISCO radius of the deviation parameter $\epsilon$ and spin of a Kerr black hole for the magnetic dipoles with negative and positive values of the magnetic coupling parameters, with $\beta= \pm 0.25$ and neutral particles. We note that when the direction of the magnetic dipole moment of the test particle aligns along the magnetic field the magnetic coupling parameter is positive, otherwise it is negative.

In Fig. 9 we provide the behavior of the ISCO radius of the magnetic dipoles around a quasi-Schwarzschild black hole in the presence and absence of an external magnetic field (blue dashed, red dot-dashed and black solid lines in the top panel of the figure) and around a rotating Kerr black hole in the absence of an external magnetic field. One may see from the top panel of the figure that an increase of the positive devia- tion parameter causes a decrease of the ISCO radius while a negative one leads to an increase. In some cases it is similar to the effect of the spin of a Kerr black hole. Moreover, positive (negative) values of the magnetic coupling parameter shift the ISCO radius outwards from (towards) the central black hole. In the bottom panel we show (compare) the effects of the deviation parameter of a quasi-Schwarzchild and the spin of a Kerr black holes for the degeneracy cases of the magnetic dipoles having the same values for the ISCO radius. One may see from the degeneracy plot that a negative deviation parameter can mimic the spin of a Kerr black hole providing the same value of the ISCO radius for corotating orbits of magnetized particles with the magnetic coupling parameter $\beta=0.25$ up to $a / M \simeq 0.3952$ while, for the particles with the parameter $\beta=0$ and $\beta=-0.25$, it mimics up to the spin 


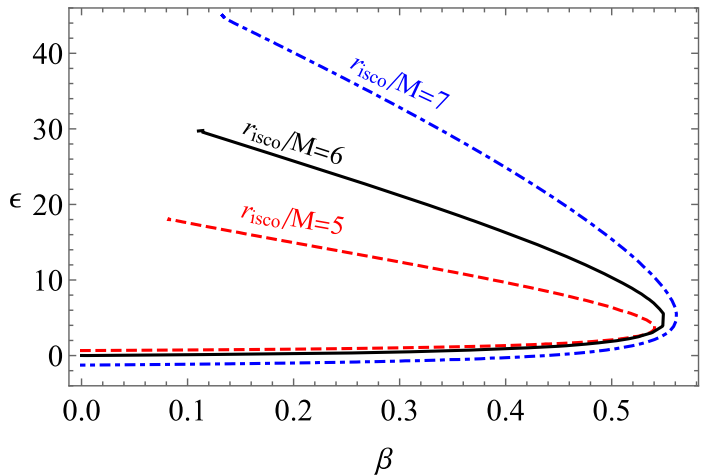

Fig. 11 Relations between the deviation parameter $\epsilon$ and magnetic coupling parameter $\beta$ for fixed values of the ISCO radius of the magnetic dipoles

value of a Kerr black hole $a \simeq 0.1984 M$ and $a \simeq 0.0537 M$, respectively.

Here, we will focus on how the magnetic coupling parameter can mimic the spin of the Kerr black hole providing the same value for the ISCO radius of the magnetic dipoles around a Schwarzschild black hole. One may easily calculate the ISCO radius for magnetic dipoles around the Schwarzschild black hole keeping the deviation parameter zero.

One can construct the degeneracy plot between the magnetic parameter in quasi-Schwarzschild metric and the rotation parameter of the Kerr one. From the right panel of Fig. 8 it is clearly seen that for fixed values of the deviation parameter $\epsilon$ the ISCO radius grows similar to the case when one increases the magnetic coupling parameter. It leads to the conclusion that this coupling parameter should only mimic the rotation parameter of a Kerr metric for retrograde orbits. One can be ensured of this from the degeneracy plot as illustrated in Fig. 10

The degeneracy between values of parameter $\epsilon$ and magnetic coupling parameter for fixed values of the ISCO radius is presented in Fig. 11. One can easily see that there are degeneracy values for the deviation parameter when the magnetic coupling parameter is fixed which provides the same value for the ISCO radius. Consequently, with the increase of the ISCO radius the range of degeneracy values of the deviation parameter increases.

\section{Conclusion}

In this work the motion of charged particles together with magnetic dipoles has been investigated to determine how well the spacetime deviation parameter $\epsilon$ and external uniform magnetic field can mimic the rotation parameter of a Kerr black hole, which is the main point of this study.
The investigation of charged particle motion has shown that the deviation parameter $\epsilon$ in the absence of an external magnetic field can mimic the rotation parameter of the Kerr spacetime up to $a \approx 0.46$ which means that the black hole is assumed to be a Kerr one; up to such a rotation parameter there can be also a static quasi-Schwarzschild one with deviation parameter up to $\epsilon \approx 0.8$. It has been also shown that the external magnetic field itself (i.e. without deviation parameter of spacetime) can mimic the rotation parameter up to $a \approx 0.88$. The combination of these two parameters can do an even better but not considerable job mimicking the rotation parameter up to $a>0.9$.

The study of the dynamics of the magnetic dipoles around a quasi-Schwarzschild black hole in the external magnetic field has shown that the maximum value of the effective potential for fixed values of the specific angular momentum of the magnetic dipoles and the deviation parameter of the spacetime around the $\mathrm{BH}$ increases with the increase of the magnetic coupling parameter. The positive deviation parameter increases the effective potential at fixed values of the angular momentum and the magnetic coupling parameter while the negative ones decrease. It is shown that there are degeneracy values of the ISCO radius of test particles at $\epsilon_{c r}>\epsilon \geq 0.35$, which may lead to two different values of the ISCO radius. Finally, we have studied how the deviation parameter mimics the spin of the Kerr $\mathrm{BH}$ providing the same values for the ISCO radius of the test particles. Since we consider magnetic dipoles as test ones, we have chosen here two different signs for the magnetic coupling parameter in the range of $\beta \in[-0.25,0.25]$ and obtained the result that when the value of the deviation parameter is in the range of $\epsilon \in(-1,1)$ it can mimic the spin of a rotating Kerr $\mathrm{BH}$ in the range $a / M \in(0.0537,0.3952)$ for the magnetic dipoles with the values of the magnetic coupling parameter $\beta \in[-0.25,0.25]$ in corotating orbits. However, the mimic values of the spin parameter in counterrotating orbits lie in the range of $a / M \in(0.0152,0.7863)$. We have pointed out that, since the magnetic coupling parameter increases the ISCO radius of the magnetic dipoles, it can mimic the spin of the Kerr BH only in corotating orbits up to its value $\beta=0.5922$. Moreover, we have shown the degeneracy relations between the magnetic coupling parameter and the deviation parameters for fixed values of the ISCO radius and found that the ISCO radius can be the same at the two different positive values of the deviation parameter for fixed values of the magnetic coupling parameter. The study performed can be applied to the dynamics of magnetized matter and neutron stars in the environment close to SMBH.

Acknowledgements This research is supported by the Uzbekistan Ministry for Innovative Development, Grants no. VA-FA-F-2-008 and no. MRB-AN-2019-29, the Innovation Program of the Shanghai Municipal Education Commission, Grant No. 2019-01-07-00-07-E00035, and the National Natural Science Foundation of China (NSFC), Grant 
no. 11973019. B.N. also acknowledges support from the China Scholarship Council (CSC), grant no. 2018DFH009013. This research is partially supported by an Erasmus+ exchange Grant between SU and NUUz. A.A. is supported by a postdoc fund through PIFI of the Chinese Academy of Sciences.

Data Availability Statement This manuscript has no associated data or the data will not be deposited. [Authors' comment:Data sharing not applicable to this article as no datasets were generated or analyzed during the current study.]

Open Access This article is licensed under a Creative Commons Attribution 4.0 International License, which permits use, sharing, adaptation, distribution and reproduction in any medium or format, as long as you give appropriate credit to the original author(s) and the source, provide a link to the Creative Commons licence, and indicate if changes were made. The images or other third party material in this article are included in the article's Creative Commons licence, unless indicated otherwise in a credit line to the material. If material is not included in the article's Creative Commons licence and your intended use is not permitted by statutory regulation or exceeds the permitted use, you will need to obtain permission directly from the copyright holder. To view a copy of this licence, visit http://creativecomm ons.org/licenses/by/4.0/.

Funded by SCOAP ${ }^{3}$.

\section{References}

1. K. Schwarzschild, Abh. Konigl. Preuss. Akad. Wissenschaften Jahre 1906,92, Berlin, 1907 1916, 189 (1916)

2. E. Newman, L. Tamburino, T. Unti, J. Math. Phys. 4, 915 (1963)

3. R.L. Zimmerman, B.Y. Shahir, Gen. Relativ. Gravit. 21, 821 (1989)

4. K. Glampedakis, S. Babak, Class. Quantum Gravity 23, 4167 (2006). arXiv:gr-qc/0510057

5. T. Johannsen, D. Psaltis, Astrophys. J. 716, 187 (2010). arXiv:1003.3415 [astro-ph.HE]

6. T. Johannsen, D. Psaltis, Phys. Rev. D 83, 124015 (2011). arXiv:1105.3191 [gr-qc]

7. T. Johannsen, Astrophys. J. 777, 170 (2013). arXiv:1501.02814 [astro-ph.HE]

8. R. Konoplya, L. Rezzolla, A. Zhidenko, Phys. Rev. D 93, 064015 (2016). arXiv:1602.02378 [gr-qc]

9. V. Cardoso, P. Pani, J. Rico, Phys. Rev. D 89, 064007 (2014). arXiv:1401.0528 [gr-qc]

10. A. Sen, Phys. Rev. Lett. 69, 1006 (1992). arXiv:hep-th/9204046

11. L. Rezzolla, A. Zhidenko, Phys. Rev. D 90, 084009 (2014). arXiv:1407.3086 [gr-qc]

12. S. Grunau, V. Kagramanova, Phys. Rev. D 83, 044009 (2011). arXiv: 1011.5399 [gr-qc]

13. A.F. Zakharov, Class. Quantum Gravity 11, 1027 (1994)

14. Z. Stuchlík, S. Hledik, Acta Phys. Slovaca 52, 363 (2002)

15. D. Pugliese, H. Quevedo, R. Ruffini, ArXiv e-prints, arXiv: 1003.2687 [gr-qc] (2010)

16. D. Pugliese, H. Quevedo, R. Ruffini, Phys. Rev. D 83, 024021 (2011a). arXiv:1012.5411 [astro-ph.HE]

17. D. Pugliese, H. Quevedo, R. Ruffini, Phys. Rev. D 83, 104052 (2011b). arXiv:1103.1807 [gr-qc]

18. M. Patil, P.S. Joshi, M. Kimura, K.-I. Nakao, Phys. Rev. D 86, 084023 (2012). arXiv:1108.0288 [gr-qc]

19. B.V. Turimov, B.J. Ahmedov, A.A. Hakimov, Phys. Rev. D 96, 104001 (2017)

20. R. Whisker, Phys. Rev. D 71, 064004 (2005). arXiv:astro-ph/0411786
21. A.S. Majumdar, N. Mukherjee, Mod. Phys. Lett. A 20, 2487 (2005). arXiv:astro-ph/0403405

22. J. Liang, Commun. Theor. Phys. 67, 407 (2017)

23. G. Li, B. Cao, Z. Feng, X. Zu, Int. J. Theor. Phys. 54, 3103 (2015). arXiv: 1506.08410 [gr-qc]

24. C. Liu, S. Chen, C. Ding, J. Jing, Phys. Lett. B 701, 285 (2011). arXiv: 1012.5126 [gr-qc]

25. V.S. Morozova, B.J. Ahmedov, Int. J. Mod. Phys. D 18, 107 (2009). arXiv:0804.2786 [gr-qc]

26. A.N. Aliev, H. Cebeci, T. Dereli, Phys. Rev. D 77, 124022 (2008). arXiv:0803.2518 [hep-th]

27. B.J. Ahmedov, A.V. Khugaev, A.A. Abdujabbarov, Astrophys. Space Sci. 337, 679 (2012). arXiv:1109.0822 [astro-ph.SR]

28. A.A. Abdujabbarov, B.J. Ahmedov, S.R. Shaymatov, A.S. Rakhmatov, Astrophys. Space Sci. 334, 237 (2011a). arXiv:1105.1910 [astro-ph.SR]

29. A.A. Abdujabbarov, B.J. Ahmedov, V.G. Kagramanova, Gen. Relativ. Gravit. 40, 2515 (2008). arXiv:0802.4349 [gr-qc]

30. C. Bambi, Rev. Mod. Phys. 89, 025001 (2017)

31. J.R. Rayimbaev, B.J. Ahmedov, N.B. Juraeva, A.S. Rakhmatov, Astrophys. Space Sci. 356, 301 (2015)

32. C. Bambi, E. Barausse, Phys. Rev. D. 84, 084034 (2011). arXiv: 1108.4740 [gr-qc]

33. S. Chen, J. Jing, Phys. Rev. D 85, 124029 (2012). arXiv: 1204.2468 [gr-qc]

34. B. Narzilloev, D. Malafarina, A. Abdujabbarov, C. Bambi, Eur. Phys. J. C 80, 784 (2020). arXiv:2003.11828 [gr-qc]

35. C. Bambi, F. Caravelli, L. Modesto, Phys. Lett. B 711, 10 (2012). arXiv:1110.2768 [gr-qc]

36. C. Bambi, Phys. Rev. D 87, 023007 (2013). arXiv:1211.2513 [grqc]

37. C. Bambi, A. Cárdenas-Avendaño, T. Dauser, J.A. García, S Nampalliwar, Astrophys. J. 842, 76 (2017). arXiv:1607.00596 [gr-qc]

38. Z. Cao, S. Nampalliwar, C. Bambi, T. Dauser, J.A. García, Phys. Rev. Lett. 120, 051101 (2018). arXiv:1709.00219 [gr-qc]

39. D. Psaltis, T. Johannsen, Astrophys. J. 745, 1 (2012). arXiv:1011.4078 [astro-ph.HE]

40. C. Liu, S. Chen, J. Jing, J. High Energy Phys. 8, 97 (2012). arXiv: 1208.1072 [gr-qc]

41. H. Chakrabarty, A.B. Abdikamalov, A.A. Abdujabbarov, C. Bambi, Phys. Rev. D 98, 024022 (2018). arXiv:1804.00461 [gr$\mathrm{qc}]$

42. B. Narzilloev, A. Abdujabbarov, C. Bambi, B. Ahmedov, Phys. Rev. D 99, 104009 (2019). arXiv:1902.03414 [gr-qc]

43. K. Haydarov, J. Rayimbaev, A. Abdujabbarov, S. Palvanov, D. Begmatova, Eur. Phys. J. C 80, 399 (2020). arXiv:2004.14868 [gr-qc]

44. K. Haydarov, A. Abdujabbarov, J. Rayimbaev, B. Ahmedov, Universe (2020). https://doi.org/10.3390/universe6030044

45. B. Narzilloev, J. Rayimbaev, A. Abdujabbarov, C. Bambi, Eur. Phys. J. C 80, 1074 (2020a). arXiv:2005.04752 [gr-qc]

46. A. Abdujabbarov, J. Rayimbaev, B. Turimov, F. Atamurotov, Phys. Dark Univ. 30, 100715 (2020a)

47. B. Turimov, J. Rayimbaev, A. Abdujabbarov, B. Ahmedov, Z.C.V. Stuchlík, Phys. Rev. D 102, 064052064052 (2020)

48. B. Narzilloev, J. Rayimbaev, S. Shaymatov, A. Abdujabbarov, B. Ahmedov, C. Bambi, Phys. Rev. D 102, 104062 (2020b). arXiv:2011.06148 [gr-qc]

49. J. Rayimbaev, A. Abdujabbarov, M. Jamil, B. Ahmedov, W.-B. Han, Phys. Rev. D 102, 084016 (2020)

50. R. Pánis, M. Kološ, Z. Stuchlík, Eur. Phys. J. C 79, 479 (2019). arXiv:1905.01186 [gr-qc]

51. M. Kološ, Z. Stuchlík, A. Tursunov, Class. Quantum Gravity 32, 165009 (2015). arXiv:1506.06799 [gr-qc] 
52. A. Tursunov, Z. Stuchlík, M. Kološ, Phys. Rev. D 93, 084012 (2016). arXiv:1603.07264 [gr-qc]

53. Z. Stuchlík, M. Kološ, Eur. Phys. J. C 76, 32 (2016). arXiv: 1511.02936 [gr-qc]

54. Z. Stuchlík, M. Kološ, J. Kovář, P. Slaný, A. Tursunov, Universe 6, $26(2020)$

55. A. Tursunov, Z. Stuchlík, M. Kološ, N. Dadhich, B. Ahmedov, Astrophys. J. 895, 14 (2020). arXiv:2004.07907 [astro-ph.HE]

56. M. Takahashi, H. Koyama, Astrophys. J. 693, 472 (2009). arXiv:0807.0277 [astro-ph]

57. O. Kopáček, V. Karas, J. Kováŕ, Z. Stuchlík, Astrophys. J. 722, 1240 (2010). arXiv:1008.4650 [astro-ph.HE]

58. O. Kopáček, V. Karas, Astrophys. J. 787, 117 (2014). arXiv: 1404.5495 [astro-ph.HE]

59. D. Li, X. Wu, Eur. Phys. J. Plus 134, 96 (2019). arXiv:1803.02119 [gr-qc]

60. M. Yi, X. Wu, Phys. Scr. 95, 085008 (2020)

61. K. Akiyama et al., Event horizon telescope. Astrophys. J. 875, L1 (2019a)

62. K. Akiyama et al., Event horizon telescope. Astrophys. J. 875, L6 (2019b)

63. The LIGO Scientific Collaboration and the Virgo Collaboration, ArXiv e-prints, arXiv:1602.03841 [gr-qc] (2016)

64. B.P. Abbott, R. Abbott, T.D. Abbott, M.R. Abernathy, F. Acernese, K. Ackley, C. Adams, T. Adams, P. Addesso, R.X. Adhikari et al., Phys. Rev. Lett. 116, 061102 (2016). arXiv:1602.03837 [gr-qc]

65. J. Levin, Phys. Rev. Lett 84, 3515 (2000). arXiv:gr-qc/9910040

66. J. Levin, Phys. Rev. D 67, 044013 (2003). arXiv:gr-qc/0010100

67. N.J. Cornish, J. Levin, Phys. Rev. Lett 89, 179001 (2002). arXiv:gr-qc/0207020

68. N.J. Cornish, J. Levin, Phys. Rev. D 68, 024004 (2003)

69. A. Buonanno, Y. Chen, Y. Pan, H. Tagoshi, M. Vallisneri, Phys. Rev. D 72, 084027 (2005). arXiv:gr-qc/0508064

70. X. Wu, Y. Xie, Phys. Rev. D 76, 124004 (2007). arXiv:1004.5057 [gr-qc]

71. X. Wu, Y. Xie, Phys. Rev. D 77, 103012 (2008). arXiv: 1004.5317 [gr-qc]

72. X. Wu, Y. Xie, Phys. Rev. D 81, 084045 (2010). arXiv:1004.4549 [gr-qc]

73. X. Wu, L. Mei, G. Huang, S. Liu, Phys. Rev. D 91, 024042 (2015)

74. X. Wu, G. Huang, Mon. Not. R. Astron. 452, 3167 (2015)

75. S.-Y. Zhong, X. Wu, S.-Q. Liu, X.-F. Deng, Phys. Rev. D 82, 124040 (2010)

76. L. Mei, X. Wu, F. Liu, Eur. Phys. J. C 73, 2413 (2013)

77. C. Bambi, D. Malafarina, L. Modesto, J. High Energy Phys. 2016, 147 (2016). arXiv:1603.09592 [gr-qc]

78. M. Zhou, Z. Cao, A. Abdikamalov, D. Ayzenberg, C. Bambi, L. Modesto, S. Nampalliwar, Phys. Rev. D 98, 024007 (2018). arXiv: 1803.07849 [gr-qc]

79. A. Tripathi, J. Yan, Y. Yang, Y. Yan, M. Garnham, Y. Yao, S. Li, Z. Ding, A.B. Abdikamalov, D. Ayzenberg, C. Bambi, T. Dauser, J.A. Garcia, J. Jiang, S. Nampalliwar, ArXiv e-prints, arXiv:1901.03064 [gr-qc] (2019)

80. C.W. Misner, K.S. Thorne, J.A. Wheeler, Gravitation, Misner 73 (W.H. Freeman, San Francisco, 1973)

81. R.M. Wald, Phys. Rev. D. 10, 1680 (1974)

82. S. Chen, M. Wang, J. Jing, J. High Energy Phys. 2016, 82 (2016). arXiv: 1604.02785 [gr-qc]

83. K. Hashimoto, N. Tanahashi, Phys. Rev. D 95, 024007 (2017). arXiv: 1610.06070 [hep-th]

84. S. Dalui, B.R. Majhi, P. Mishra, Phys. Lett. B 788, 486 (2019). arXiv: 1803.06527 [gr-qc]

85. W. Han, Gen. Relativ. Gravit. 40, 1831 (2008). arXiv:1006.2229 [gr-qc]

86. A.P.S. de Moura, P.S. Letelier, Phys. Rev. E 61, 6506 (2000). arXiv:chao-dyn/9910035
87. V.S. Morozova, L. Rezzolla, B.J. Ahmedov, Phys. Rev. D 89, 104030 (2014). arXiv:1310.3575 [gr-qc]

88. A. Jawad, F. Ali, M. Jamil, U. Debnath, Commun. Theor. Phys. 66, 509 (2016). arXiv:1610.07411 [gr-qc]

89. S. Hussain, M. Jamil, Phys. Rev. D 92, 043008 (2015). arXiv: 1508.02123 [gr-qc]

90. M. Jamil, S. Hussain, B. Majeed, Eur. Phys. J. C 75, 24 (2015). arXiv:1404.7123 [gr-qc]

91. S. Hussain, I. Hussain, M. Jamil, Eur. Phys. J. C 74, 210 (2014)

92. G.Z. Babar, M. Jamil, Y.-K. Lim, Int. J. Mod. Phys. D 25, 1650024 (2016). arXiv:1504.00072 [gr-qc]

93. M. Bañados, J. Silk, S.M. West, Phys. Rev. Lett. 103, 111102 (2009)

94. B. Majeed, M. Jamil, Int. J. Mod. Phys. D 26, 1741017 (2017). arXiv:1705.04167 [gr-qc]

95. A. Zakria, M. Jamil, J. High Energy Phys. 2015, 147 (2015). arXiv:1501.06306 [gr-qc]

96. I. Brevik, M. Jamil, Int. J. Geom Methods Mod. Phys. 16, 1950030 (2019). arXiv:1901.00002 [gr-qc]

97. M. De Laurentis, Z. Younsi, O. Porth, Y. Mizuno, L. Rezzolla, Phys. Rev. D 97, 104024 (2018). arXiv:1712.00265 [gr-qc]

98. S.R. Shaymatov, B.J. Ahmedov, A.A. Abdujabbarov, Phys. Rev. D 88, $024016(2013)$

99. F. Atamurotov, B. Ahmedov, S. Shaymatov, Astrophys. Space Sci. 347, 277 (2013)

100. B. Narzilloev, J. Rayimbaev, S. Shaymatov, A. Abdujabbarov, B. Ahmedov, C. Bambi, Phys. Rev. D 102, 044013 (2020c). arXiv:2007.12462 [gr-qc]

101. M. Kološ, A. Tursunov, Z. Stuchlík, Eur. Phys. J. C. 77, 860 (2017). arXiv:1707.02224 [astro-ph.HE]

102. J. Kovář, O. Kopáček, V. Karas, Z. Stuchlík, Class. Quantum Gravity 27, 135006 (2010). arXiv:1005.3270 [astro-ph.HE]

103. J. Kováŕ, P. Slaný, C. Cremaschini, Z. Stuchlík, V. Karas, A. Trova, Phys. Rev. D 90, 044029 (2014). arXiv:1409.0418 [gr-qc]

104. A.N. Aliev, D.V. Gal'tsov, Sov. Phys. Uspekhi 32, 75 (1989)

105. A.N. Aliev, N. Özdemir, Mon. Not. R. Astron. Soc. 336, 241 (2002). arXiv:gr-qc/0208025

106. A.N. Aliev, D.V. Galtsov, V.I. Petukhov, Astrophys. Space Sci. 124, 137 (1986)

107. V.P. Frolov, P. Krtouš, Phys. Rev. D 83, 024016 (2011). arXiv: 1010.2266 [hep-th]

108. V.P. Frolov, Phys. Rev. D 85, 024020 (2012). arXiv:1110.6274 [gr-qc]

109. Z. Stuchlík, J. Schee, A. Abdujabbarov, Phys. Rev. D 89, 104048 (2014)

110. S. Shaymatov, F. Atamurotov, B. Ahmedov, Astrophys. Space Sci. 350, 413 (2014)

111. A. Abdujabbarov, B. Ahmedov, Phys. Rev. D 81, 044022 (2010). arXiv:0905.2730 [gr-qc]

112. A. Abdujabbarov, B. Ahmedov, A. Hakimov, Phys. Rev. D 83, 044053 (2011b). arXiv:1101.4741 [gr-qc]

113. V. Karas, J. Kovar, O. Kopacek, Y. Kojima, P. Slany, Z. Stuchlik, in American Astronomical Society Meeting Abstracts \#220, American Astronomical Society Meeting Abstracts, vol. 220 (2012) p. 430.07

114. S. Shaymatov, M. Patil, B. Ahmedov, P.S. Joshi, Phys. Rev. D 91, 064025 (2015). arXiv:1409.3018 [gr-qc]

115. J. Rayimbaev, B. Turimov, F. Marcos, S. Palvanov, A. Rakhmatov, Mod. Phys. Lett. A 35, 2050056 (2020)

116. B. Turimov, B. Ahmedov, A. Abdujabbarov, C. Bambi, Phys. Rev. D 97, 124005 (2018). arXiv:1805.00005 [gr-qc]

117. S. Shaymatov, J. Vrba, D. Malafarina, B. Ahmedov, Z. Stuchlík, Phys. Dark Universe 30, 100648 (2020a). arXiv:2005.12410 [grqc]

118. S. Shaymatov, Int. J. Mod. Phys. Conf. Ser. 49, 1960020 (2019) 
119. J. Rayimbaev, B. Turimov, B. Ahmedov, Int. J. Mod. Phys. D 28, 1950128-209 (2019a)

120. J. Rayimbaev, P. Tadjimuratov, Phys. Rev. D 102, 024019 (2020)

121. J. Rayimbaev, B. Turimov, S. Palvanov, Int. J. Mod. Phys. Conf. Ser. 49, 1960019-209 (2019)

122. S. Shaymatov, D. Malafarina, B. Ahmedov, ArXiv e-prints, arXiv:2004.06811 [gr-qc] (2020)

123. A. Hakimov, A. Abdujabbarov, B. Narzilloev, Int. J. Mod. Phys. A 32, 1750116 (2017)

124. V. Karas, D. Vokrouhlický, Gen. Relativ. Gravit. 24, 729 (1992)

125. Y. Nakamura, T. Ishizuka, Astrophys. Space Sci. 210, 105 (1993)

126. F. de Felice, F. Sorge, Class. Quantum Gravity 20, 469 (2003)

127. F. de Felice, F. Sorge, S. Zilio, Class. Quantum Gravity 21, 961 (2004)

128. J.R. Rayimbaev, Astrophys. Space Sci. 361, 288 (2016)

129. T. Oteev, A. Abdujabbarov, Z. Stuchlík, B. Ahmedov, Astrophys. Space Sci. 361, 269 (2016)

130. B. Toshmatov, A. Abdujabbarov, B. Ahmedov, Z. Stuchlík, Astrophys. Space Sci. 360, 19 (2015)

131. A. Abdujabbarov, B. Ahmedov, O. Rahimov, U. Salikhbaev, Phys. Scr. 89, 084008 (2014)
132. O.G. Rahimov, A.A. Abdujabbarov, B.J. Ahmedov, Astrophys. Space Sci. 335, 499 (2011). arXiv:1105.4543 [astro-ph.SR]

133. O.G. Rahimov, Mod. Phys. Lett. A 26, 399 (2011). arXiv: 1012.1481 [gr-qc]

134. J. Vrba, A. Abdujabbarov, M. Kološ, B. Ahmedov, Z. Stuchlík, J. Rayimbaev, Phys. Rev. D 101, 124039 (2020)

135. A. Abdujabbarov, J. Rayimbaev, B. Turimov, F. Atamurotov, Phys. Dark Univ. 30, 100715 (2020b)

136. K. Mori, E.V. Gotthelf, S. Zhang, H. An, F.K. Baganoff, N.M. Barrière, A.M. Beloborodov, S.E. Boggs, F.E. Christensen, W.W. Craig, F. Dufour, B.W. Grefenstette, C.J. Hailey, F.A. Harrison, J. Hong, V.M. Kaspi, J.A. Kennea, K.K. Madsen, C.B. Markwardt, M. Nynka, D. Stern, J.A. Tomsick, W.W. Zhang, Astron. J. Lett. 770, L23 (2013). arXiv:1305.1945 [astro-ph.HE]

137. J.M. Bardeen, W.H. Press, S.A. Teukolsky, Astrophys. J. 178, 347 (1972) 